\title{
Oxygenation Index in the First 24 Hours after the Diagnosis of Acute Respiratory Distress Syndrome as a Surrogate Metric for Risk Stratification in Children
}

\author{
Soo Yeon Kim, Byuhree Kim', Sun Ha Choi ${ }^{1}$, Jong Deok Kim ${ }^{1,2}$, In Suk Sol ${ }^{1}$, Min Jung Kim ${ }^{1}$, Yoon Hee Kim¹, \\ Kyung Won Kim', Myung Hyun Sohn', Kyu-Earn Kim ${ }^{1,3}$ \\ 'Department of Pediatrics, Yonsei University College of Medicine, Seoul, ${ }^{2}$ Department of Pediatrics, Hanyang University College of Medicine, Seoul; ${ }^{3}$ Sowha \\ Children's Hospital, Seoul, Korea
}

Background: The diagnosis of pediatric acute respiratory distress syndrome (PARDS) is a pragmatic decision based on the degree of hypoxia at the time of onset. We aimed to determine whether reclassification using oxygenation metrics 24 hours after diagnosis could provide prognostic ability for outcomes in PARDS.

Methods: Two hundred and eighty-eight pediatric patients admitted between January 1, 2010 and January 30, 2017, who met the inclusion criteria for PARDS were retrospectively analyzed. Reclassification based on data measured 24 hours after diagnosis was compared with the initial classification, and changes in pressure parameters and oxygenation were investigated for their prognostic value with respect to mortality.

Results: PARDS severity varied widely in the first 24 hours; $52.4 \%$ of patients showed an improvement, 35.4\% showed no change, and 12.2\% either showed progression of PARDS or died. Multivariate analysis revealed that mortality risk significantly increased for the severe group, based on classification using metrics collected 24 hours after diagnosis (adjusted odds ratio, 26.84; 95\% confidence interval [Cl], 3.43 to 209.89; $P=0.002$ ). Compared to changes in pressure variables (peak inspiratory pressure and driving pressure), changes in oxygenation (arterial partial pressure of oxygen to fraction of inspired oxygen) over the first 24 hours showed statistically better discriminative power for mortality (area under the receiver operating characteristic curve, $0.701 ; 95 \% \mathrm{Cl}, 0.636$ to $0.766 ; \mathrm{P}<0.001)$.

Conclusions: Implementation of reclassification based on oxygenation metrics 24 hours after diagnosis effectively stratified outcomes in PARDS. Progress within the first 24 hours was significantly associated with outcomes in PARDS, and oxygenation response was the most discernable surrogate metric for mortality.

Key Words: acute respiratory distress syndrome; mortality; pediatrics; risk assessment

\section{INTRODUCTION}

Acute respiratory distress syndrome (ARDS) refers to a condition of acute lung injury and inflammation that leads to hypoxic respiratory failure. Although there is general agreement that ARDS can affect people of all ages [1], the original and revised definitions of ARDS (i.e., the American-European Consensus Conference definition in 1994 [2] and the Berlin defini-

\section{Original Article}

Received: April 20, 2018

Revised: August 10, 2018

Accepted: August 27, 2018

Corresponding author

Kyung Won Kim

Department of Pediatrics, Severance Children's Hospital, Yonsei University College of Medicine, 50-1 Yonsei-ro, Seodaemun-gu, Seoul 03722, Korea Tel: +82-2-2228-2050

Fax: +82-2-393-9118

E-mail: kwkim@yuhs.ac

Copyright () 2018 The Korean Society of Critical Care Medicine

This is an Open Access article distributed under the terms of Creative Attributions Non-Commercial License (http:// creativecommons.org/li-censes/by-nc/4.0/) which permits unrestricted noncommercial use, distribution, and reproduction in any medium, provided the original work is properly cited. 
tion in 2012 [3]) did not include pediatric considerations. As the pediatric population exhibits a distinctive epidemiology and prognosis [4], as well as intrinsic differences in respiratory physiology [5], the Pediatric Acute Lung Injury Consensus Conference (PALICC) established new definitions for pediatric ARDS (PARDS) in 2015 [6]. The key differences included in these PALICC criteria are the use of an oxygenation index (OI) for severity stratification instead of the ratio of the arterial partial oxygen pressure to the fraction of inspired oxygen $\left(\mathrm{PaO}_{2} /\right.$ $\mathrm{FiO}_{2}$ ), allowance for pulse oximetry-based metrics (e.g., oxygenation saturation index [OSI]) when $\mathrm{PaO}_{2}$ is not available, less stringent chest radiograph standards, and a broader scope by including subjects with chronic lung disease and cyanotic heart disease [6].

The incidence of PARDS is reported as $1 \%-4 \%$ of pediatric intensive care unit (ICU) admissions [7,8]. Despite improvements in the quality of intensive care, PARDS still is a major challenge in the pediatric ICU due to mortality as high as $30 \%$ $[7,9]$. As most treatment strategies for PARDS have been extrapolated from evidence gathered in adult studies, there is a growing need for interventional trials focused on pediatric populations. Likewise, risk stratification and identification of high-risk cases of PARDS have been attempted to better understand patient populations and optimize the quality of care.

Although the above definitions use oxygenation metrics as a primary indicator for lung injury, there remains a lack of guidance regarding when clinicians should acquire the metrics for PARDS risk stratification. With regard to $\mathrm{PaO}_{2} / \mathrm{FiO}_{2}$, OI and OSI are influenced by ventilator settings [10-12] and vary according to the disease progression; thus, the timing of measuring these metrics should be considered during risk stratification. Notably, several recent reports $[8,13,14]$ have shown that mortality discrimination in PARDS is improved with the use of oxygenation metrics within the first 24 hours after onset relative to using initial metrics.

In this study, we aimed to determine whether reclassification using oxygenation metrics 24 hours after diagnosis of PARDS would have prognostic ability in an Asian cohort. Respiratory variables to be used as early prediction factors for risk stratification in PARDS were also investigated.

\section{MATERIALS AND METHODS}

\section{Patient Selection}

A total of 1,101 patients aged 1 month to 19 years who were treated in the medical ICU of a medical ICU of Severance Hospital between January 1, 2010 and January 30, 2017 were screen-

\section{KEY MESSAGES}

- Pediatric acute respiratory distress syndrome (PARDS) severity varied widely in the first 24 hours and the progress within this early timeframe was significantly associated with mortality and ventilator-free days.

- Improvement in oxygenation in the first 24 hours after the diagnosis was the most discernable predictor for outcomes in PARDS.

ed for inclusion in this study. All pediatric patients admitted to the medical ICU during this period were identified using an electronic database search, and two of the authors (SYK and BK) identified patients who fulfilled the following eligibility criteria: (1) acute respiratory failure requiring invasive mechanical ventilation, (2) at least one documented arterial bloodgas result at the time of PARDS diagnosis, (3) diagnosis of PARDS according to the PALICC criteria, and (4) age older than 1 month. The exclusion criteria were as follows: (1) respiratory failure due to cardiac failure or fluid overload, (2) congenital cyanotic heart disease, and (3) initiation of mechanical ventilation for more than 7 days before fulfilling OI $\geq 4$. For the patients who met the inclusion criteria, clinical data were collected from the electronic medical records and analyzed retrospectively. This study was approved by the Institutional Review Board of the Institutional Review Board of Severance Hospital (IRB No. 4-2013-0207), and the informed consent requirement was waived.

\section{Data Collection and Variable Definitions}

Demographics and clinical data, including PARDS etiology, comorbidity, Pediatric Risk of Mortality (PRISM) III score, clinical progress, and outcomes, were assessed. The oxygenation metrics obtained were $\mathrm{PaO}_{2} / \mathrm{FiO}_{2}$ ratio, OI [(mean airway pressure $\left.\times \mathrm{FiO}_{2} \times 100\right) / \mathrm{PaO}_{2}$ ], and OSI [(mean airway pressure $\left.\times \mathrm{FiO}_{2} \times 100\right) / \mathrm{SpO}_{2}$ ]. All patients were classified according to the PALICC criteria [15] using the oxygenation metrics at the time of diagnosis and 24 hours afterward as follows: (1) mild PARDS, $4 \leq$ OI $<8$ or $5 \leq$ OSI $<7.5$, (2) moderate PARDS, $8 \leq$ OI $<16$ or $7.5 \leq$ OSI $<12.3$, and (3) severe PARDS, OI $\geq 16$ or OSI $\geq 12.3$. Data regarding mechanical ventilation, including ventilator mode, peak inspiratory pressure (PIP), positive endexpiratory pressure (PEEP), mean airway pressure, tidal volume (TV), and $\mathrm{FiO}_{2}$, were recorded at the time of PARDS diagnosis and 24 hours later. Driving pressure was defined as the difference between the PIP and PEEP. Changes in these variables over the first 24 hours $\left(\Delta \mathrm{PaO}_{2} / \mathrm{FiO}_{2}, \Delta\right.$ driving pressure, and $\left.\Delta \mathrm{PIP}\right)$ 
were also determined.

In our institution, general patient management in cases of progressive respiratory failure is performed using mechanical ventilation strategies with a minimal PEEP of $5 \mathrm{cmH}_{2} \mathrm{O}$, escalation to $8-10 \mathrm{cmH}_{2} \mathrm{O}$ in cases of inability to wean $\mathrm{FiO}_{2}$ below 0.60 , and attempts to maintain PIP below $30-35 \mathrm{cmH}_{2} \mathrm{O}$ with TV $<5-8 \mathrm{~mL} / \mathrm{kg}$. Although there is no specific target $\mathrm{PaO}_{2}$, a $\mathrm{PaO}_{2}$ of at least $60 \mathrm{mmHg}$ is typically accepted in cases if the hemodynamics and tissue oxygenation appear adequate. Decelerating flow with either pressure-regulated volume control or pressure control mode of ventilation was used in the majority of cases. As both oxygenation $[13,16]$ and driving pressure [17] are associated with mortality in ARDS, pressure parameters (e.g., PIP and driving pressure) and oxygenation were investigated for their prognostic ability.

The reported outcomes are ICU mortality and ventilator-free days (VFDs) at 28 days postdiagnosis. All instances of "ventilation" in this study indicate invasive ventilation; noninvasive support was not counted toward the ventilator days. VFDs were determined for survivors by subtracting the total duration of ventilation from 28 days. Patients who required $\geq 28$ ventilator days and all ICU non-survivors were designated as $\mathrm{VFD}=0$. This composite endpoint, which incorporates both mortality and length of ventilation by penalizing non-survivors, is among the most commonly used surrogate endpoints in recent PARDS studies [18].

\section{Statistical Analysis}

The data are expressed as either percentages or medians with interquartile range (IQR). Categorical variables were analyzed using the chi-square test or Fisher exact test, and continuous variables were compared using the Mann-Whitney U-test. The main outcomes of PARDS were mortality and VFDs at day 28; VFDs were dichotomized into 0 days versus $>0$ days and into $\leq 14$ days vs. $>14$ days. Multivariate logistic regression was performed to test for associations between severity classifications and main outcomes, adjusting for age, sex, PRISM III score, comorbidity, and PARDS etiology. Initial progress during the first 24 hours was divided into maintaining or worsening severity versus improving severity, which were evaluated for associations with the outcomes. Receiver operating characteristic (ROC) analysis was employed to evaluate the discri-

Table 1. Demographics and baseline characteristics of the study population

\begin{tabular}{|c|c|c|c|c|}
\hline Variable & Total $(n=288)$ & Survived $(n=176)$ & Died $(n=112)$ & P-value \\
\hline Sex (male:female) & 169:119 & $100: 76$ & $69: 43$ & $<0.001$ \\
\hline Age (yr) & $3.7(1.3-10.5)$ & $2.5(1.0-8.3)$ & $6.0(2.0-11.9)$ & 0.495 \\
\hline PRISM III score & $9.0(5.0-14.5)$ & $6.5(3.0-11.0)$ & $14.0(9.0-20.0)$ & $<0.001$ \\
\hline Comorbidity in ICU at admission & & & & $<0.001$ \\
\hline Oncology & $71(45.5)$ & $17(20.0)$ & $54(76.1)$ & \\
\hline Neurology & $69(44.2)$ & $56(65.9)$ & 13 (18.3) & \\
\hline Genetic syndrome & $16(10.3)$ & $12(14.1)$ & $4(5.6)$ & \\
\hline PARDS etiology & & & & $<0.001$ \\
\hline Infectious pneumonia & $182(63.2)$ & 130 (73.9) & $52(46.4)$ & \\
\hline Aspiration pneumonia & $33(11.5)$ & $23(13.1)$ & $10(8.9)$ & \\
\hline Sepsis & $61(21.2)$ & $17(9.7)$ & 44 (39.3) & \\
\hline Initial PALICC grade & & & & $<0.001$ \\
\hline Mild: moderate : severe & 121:99:68 & $90: 63: 23$ & $31: 36: 45$ & \\
\hline Progress over the first 24 hours & & & & $<0.001$ \\
\hline Improvement in severity & $151(52.4)$ & $118(67.0)$ & $33(29.5)$ & \\
\hline \multicolumn{5}{|l|}{ Outcome } \\
\hline ICU length of stay (day) & $11.0(5.0-21.0)$ & $11.0(7.0-21.0)$ & $7.0(2.0-22.0)$ & 0.002 \\
\hline Ventilator duration (day) & $9.0(5.0-20.0)$ & $9.0(6.0-18.0)$ & 7.5 (3.0-23.0) & 0.065 \\
\hline VFDs at day 28 & 139 (48.3) & 27 (15.3) & $112(100.0)$ & $<0.001$ \\
\hline
\end{tabular}

Values are presented as median (interquartile range) or number (\%).

PRISM: Pediatric Risk of Mortality; ICU: intensive care unit; PARDS: pediatric acute respiratory distress syndrome; PALICC: Pediatric Acute Lung Injury Consensus Conference; VFD: ventilator-free day. 
minative ability of each classification at different time points for mortality and VFDs using the area under the curve with 95\% confidence intervals (CIs); comparisons between the two curves were made using DeLong's test.

All statistical analyses were performed with R software version 3.2.2 (R Foundation, Vienna, Austria) and IBM SPSS version 23.0 (IBM Corp., Armonk, NY, USA). Statistical significance was defined as $\mathrm{P}<0.05$.

\section{RESULTS}

\section{Characteristics of Study Population}

A total of 288 pediatric patients who met the inclusion criteria were included in this analysis. The characteristics of the overall study population and of each group, stratified according to survival status, are summarized in Table 1. Chronic comorbidities were present in 127 patients (44.1\%); the most common conditions were oncologic (45.5\%) and neurologic (44.2\%). Infectious pneumonia $(63.2 \%)$ and sepsis $(21.2 \%)$ composed the majority of PARDS triggers. The median length of ICU stay was 11 days (IQR, 5 to 21 days), the median duration of mechanical ventilation was 9 days (IQR, 5 to 20 days), and the overall mortality rate was $38.9 \%$.

\section{PARDS Severity Changes during the First 24 Hours}

At initial diagnosis, 121 (42.0\%), 99 (34.4\%), and 68 (23.6\%) patients met the criteria for the mild, moderate, and severe categories, respectively (Table 1). Twenty-four hours later, 25

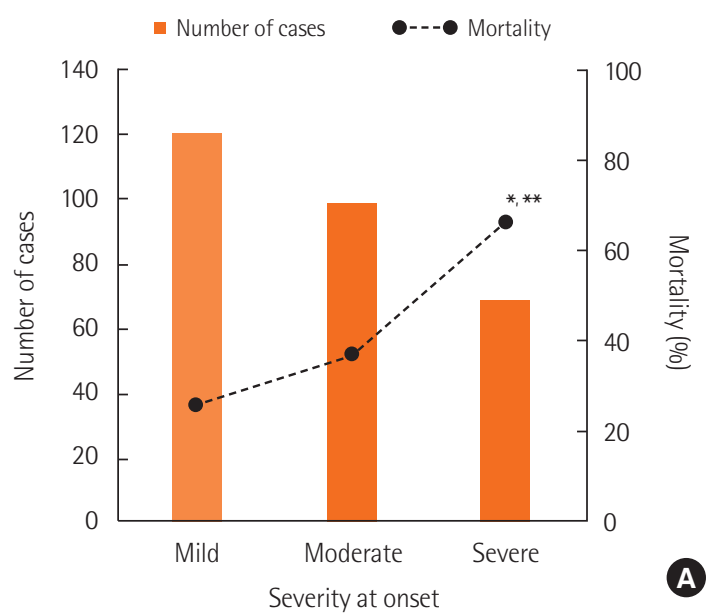

patients had died, and 187 out of the remaining 263 patients (71.1\%) continued to meet the PARDS criteria; of those, 93 cases (49.7\%) were mild, 51 (27.3\%) were moderate, and 43 (23.0\%) were severe. In the entire cohort, 151 patients $(52.4 \%)$ improved in severity or recovered from PARDS, 102 (35.4\%) maintained a stable presentation of the disease, and $35(12.2 \%)$ either exhibited aggravated disease severity or died within the first 24 hours. There were no intergroup differences according to initial PARDS severity with respect to changes in severity (data not shown).

\section{Association of Classification at Different Time Points with Outcomes in PARDS}

The classifications of the patients into severity groups at the time of PARDS diagnosis and 24 hours afterward are depicted in Figure 1. Both classifications demonstrated a stepwise increase in mortality with increasing disease severity: mild PARDS ( $n=121$; mortality, 25.6\%), moderate PARDS ( $n=99,36.4 \%$ ), and severe PARDS ( $\mathrm{n}=68,66.2 \%)$ according to metrics at diagnosis; mild PARDS ( $\mathrm{n}=93,30.1 \%)$, moderate PARDS ( $\mathrm{n}=51$, $41.2 \%$ ), and severe PARDS ( $\mathrm{n}=43,88.4 \%$ ) according to metrics at 24 hours. Significant differences in mortality were noted with severe PARDS compared to either mild or moderate PARDS (mild vs. severe PARDS, $\mathrm{P}<0.001$; moderate vs. severe PARDS, $\mathrm{P}<0.001$ ) in both classifications. Multivariate logistic regression analysis showed that the risk for mortality significantly increased for the severe group under both classifications (adjusted odds ratio [OR], 7.16; 95\% CI, 2.02 to $25.31 ; \mathrm{P}=0.002$

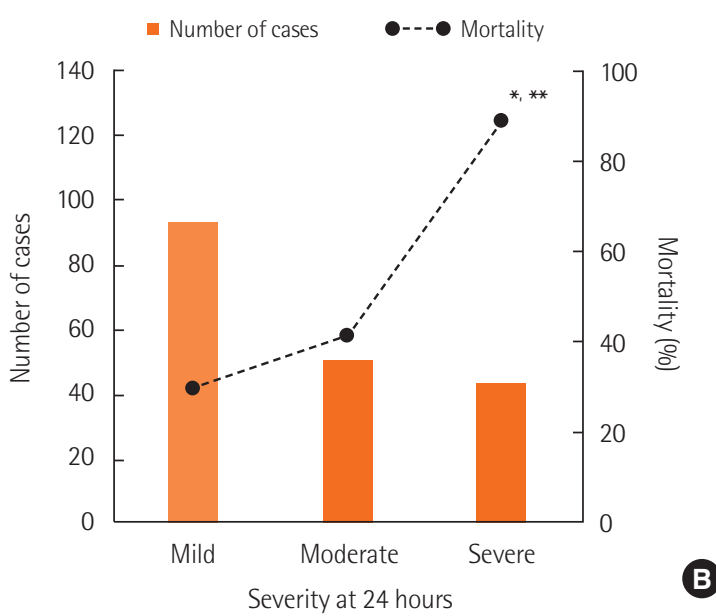

Figure 1. Classification of patients into Pediatric Acute Lung Injury Consensus Conference oxygenation categories based on metrics at the time of diagnosis (A) and 24 hours afterward (B). Both classification methods demonstrated a stepwise increase in mortality with increasing disease severity: mild PARDS $(n=121$, mortality $=25.6 \%)$, moderate PARDS $(n=99,36.4 \%)$, and severe PARDS $(n=68,66.2 \%)$ according to metrics at diagnosis; mild PARDS $(n=93,30.1 \%)$, moderate PARDS $(n=51,41.2 \%)$, and severe PARDS $(n=43,88.4 \%)$ according to metrics at 24 hours later. PARDS: pediatric acute respiratory distress syndrome. ${ }^{*}$ Mild PARDS vs. severe PARDS: P<0.001; ${ }^{* *}$ Moderate PARDS vs. severe PARDS: $\mathrm{P}<0.001$. 
Table 2. Multivariate regression analysis of association of classification at different time points with outcomes in PARDS

\begin{tabular}{|c|c|c|c|c|c|c|}
\hline \multirow{2}{*}{ Variable } & \multicolumn{3}{|c|}{ Classification at diagnosis } & \multicolumn{3}{|c|}{ Classification at 24 hours } \\
\hline & Adjusted OR & $95 \% \mathrm{Cl}$ & P-value & Adjusted OR & $95 \% \mathrm{Cl}$ & P-value \\
\hline \multicolumn{7}{|l|}{ Mortality } \\
\hline \multicolumn{7}{|c|}{ Mild (reference) } \\
\hline Moderate & 1.80 & $0.67-4.84$ & 0.241 & 2.20 & $0.53-9.18$ & 0.278 \\
\hline Severe & 7.16 & $2.02-25.31$ & 0.002 & 26.84 & $3.43-209.89$ & 0.002 \\
\hline \multicolumn{7}{|c|}{$\mathrm{VFD}=0$ at day 28} \\
\hline \multicolumn{7}{|c|}{ Mild (reference) } \\
\hline Moderate & 1.73 & $0.73-4.11$ & 0.213 & 1.58 & $0.41-6.16$ & 0.507 \\
\hline Severe & 4.56 & $1.47-14.19$ & 0.009 & 10.04 & $1.77-57.02$ & 0.009 \\
\hline \multicolumn{7}{|c|}{ VFD $\leq 14$ at day 28} \\
\hline \multicolumn{7}{|c|}{ Mild (reference) } \\
\hline Moderate & 0.97 & $0.42-2.23$ & 0.938 & 0.65 & $0.14-2.91$ & 0.569 \\
\hline Severe & 8.29 & $1.70-40.46$ & 0.009 & 6.51 & $0.63-67.16$ & 0.116 \\
\hline
\end{tabular}

ORs were adjusted for age, sex, PRISM III score, comorbidities, and PARDS etiology.

PARDS: pediatric acute respiratory distress syndrome; OR: odds ratio; Cl: confidence interval; VFD: ventilator-free day; PRISM: Pediatric Risk of Mortality.

Table 3. Multivariate regression analysis of association of initial progress with outcomes in PARDS

\begin{tabular}{lccc}
\hline Variable & Adjusted OR & $95 \% \mathrm{Cl}$ & P-value \\
\hline Mortality & & & \\
Improvement (reference) & & & \\
Maintain or aggravation & 4.52 & $1.84-11.08$ & 0.001 \\
VFD $=0$ at day 28 & & & \\
Improvement (reference) & & & \\
Maintain or aggravation & 4.62 & $2.00-10.70$ & $<0.001$ \\
VFD $\leq 14$ at day 28 & & & \\
Improvement (reference) & & & \\
Maintain or aggravation & 3.32 & $1.43-7.73$ & 0.005 \\
\hline
\end{tabular}

ORs were adjusted for age, sex, PRISM III score, comorbidities, and PARDS etiology.

PARDS: pediatric acute respiratory distress syndrome; $\mathrm{OR}$ : odds ratio; $\mathrm{Cl}$ : confidence interval; VFD: ventilator-free day; PRISM: Pediatric Risk of Mortality.

for severe group at diagnosis, and adjusted OR, 26.84; 95\% CI, 3.43 to 209.89; $\mathrm{P}=0.002$ for severe group at 24 hours). Similar results were found for patients having no VFDs at day 28 (Table 2). Subsequent ROC analysis demonstrated no differences in the discriminative ability of either mortality or VFDs between both classifications (Supplementary Figure 1).

\section{Associations of Initial Progress with Outcomes}

Initial progress was divided into maintaining or worsening severity versus improving severity. Survivors exhibited a signifi-
Table 4. Discriminative ability of respiratory variables for mortality and VFD

\begin{tabular}{lccc}
\hline Variable & $\begin{array}{c}\text { Area under } \\
\text { the curve }\end{array}$ & $95 \% \mathrm{Cl}$ & P-value \\
\hline Mortality & & & \\
$\Delta \mathrm{PaO}_{2} / \mathrm{FiO}_{2}$ & 0.701 & $0.636-0.766$ & $<0.001$ \\
$\Delta$ Driving pressure & 0.570 & $0.498-0.641$ & $<0.001$ \\
$\Delta \mathrm{PIP}$ & 0.583 & $0.511-0.656$ & $<0.001$ \\
$\mathrm{VFD}=0$ at day 28 & & & \\
$\Delta \mathrm{PaO}_{2} / \mathrm{FiO}_{2}$ & 0.658 & $0.592-0.725$ & $<0.001$ \\
$\Delta \mathrm{Driving}$ pressure & 0.554 & $0.486-0.623$ & $<0.001$ \\
$\Delta \mathrm{PIP}$ & 0.578 & $0.509-0.646$ & $<0.001$ \\
$\mathrm{VFD} \leq 14$ at day 28 & & & \\
$\Delta \mathrm{PaO}_{2} / \mathrm{FiO}_{2}$ & 0.613 & $0.543-0.683$ & $<0.001$ \\
$\Delta \mathrm{Driving}$ pressure & 0.584 & $0.516-0.652$ & $<0.001$ \\
$\Delta \mathrm{PIP}$ & 0.611 & $0.545-0.678$ & $<0.001$ \\
\hline
\end{tabular}

VFD: ventilator-free day; $\mathrm{Cl}$ : confidence interval; $\triangle \mathrm{PaO}_{2} / \mathrm{FiO}_{2}$ : changes in arterial partial pressure of oxygen to fraction of inspired oxygen for the first 24 hours; $\Delta$ Driving pressure: changes in driving pressure for the first 24 hours; $\triangle \mathrm{PIP}$ : changes in peak inspiratory pressure for the first 24 hours.

cantly higher proportion of subjects with improved severity during the first 24 hours compared with non-survivors $(67.0 \%$ and $29.5 \%$, respectively; $\mathrm{P}<0.001$ ) (Table 1 ). Logistic regression analysis revealed an association between initial progress over the first 24 hours with PARDS outcomes after adjustment for potential covariates such as age, sex, PRISM III score, co- 
Table 5. Multivariate regression analysis of association of initial oxygenation response with outcomes in PARDS

\begin{tabular}{lccr}
\hline Variable & Adjusted OR & $95 \% \mathrm{Cl}$ & P-value \\
\hline Maintained or aggravated severity & 1.54 & $1.31-1.80$ & $<0.001$ \\
Mortality & 1.09 & $1.03-1.15$ & 0.002 \\
VFD $=0$ at day 28 & 1.05 & $1.00-1.09$ & 0.031 \\
VFD $\leq 14$ at day 28 & 1.02 & $0.99-1.06$ & 0.249 \\
\hline
\end{tabular}

ORs were adjusted for age, sex, PRISM III score, comorbidities, PARDS etiology, and initial PALICC severity classification.

PARDS: pediatric acute respiratory distress syndrome; OR: odds ratio; $\mathrm{Cl}$ : confidence interval; VFD: ventilator-free day; PRISM: Pediatric Risk of Mortality; PALICC: Pediatric Acute Lung Injury Consensus Conference.

morbidity, and PARDS etiology. Maintaining or worsening severity was significantly associated with mortality (adjusted $\mathrm{OR}, 4.52 ; 95 \% \mathrm{CI}, 1.84$ to $11.08 ; \mathrm{P}=0.001)$ and $\mathrm{VFD}=0$ at day 28 (adjusted OR, 4.62; 95\% CI, 2.00 to 10.70; $\mathrm{P}<0.001$ ) (Table 3 ).

\section{Discriminative and Predictive Ability of Early Oxygenation Response}

Based on the ROC analysis, $\Delta \mathrm{PaO}_{2} / \mathrm{FiO}_{2}, \Delta$ driving pressure, and $\triangle \mathrm{PIP}$ all discriminated mortality and VFD, but the AUC of $\triangle \mathrm{PaO}_{2} / \mathrm{FiO}_{2}$ was consistently higher than the AUCs of the other factors (Table 4). For mortality, the AUC of $\Delta \mathrm{PaO}_{2} / \mathrm{FiO}_{2}$ was statistically higher compared to that of either $\Delta$ driving pressure or $\triangle$ PIP (Supplementary Figure 2), indicating superior predictive validity. Regression analysis confirmed that increasing $\mathrm{PaO}_{2} / \mathrm{FiO}_{2}$ was significantly associated with increased mortality and decreased VFD (Table 5). After adjusting for age, sex, PRISM III score, comorbidities, PARDS etiology, and initial PALICC oxygenation severity, an OR of 1.09 was determined for the odds of death with each increase of 10 in $\mathrm{PaO}_{2} /$ $\mathrm{FiO}_{2}(\mathrm{P}=0.002)$ over the first 24 hours (95\% CI, 1.03 to 1.15). Similarly, an increase of 10 in $\mathrm{PaO}_{2} / \mathrm{FiO}_{2}$ resulted in an OR of 1.05 for VFD $=0$ at day 28 ( $95 \% \mathrm{CI}, 1.00$ to $1.09 ; \mathrm{P}=0.031)$ (Table 5).

\section{DISCUSSION}

In this study, PARDS severity changed frequently in the first 24 hours after diagnosis. Reclassification 24 hours after diagnosis provided similar effectiveness in outcome stratification of PARDS subjects compared with classification by using the initial metrics. Notably, progress within the first 24 hours was significantly associated with mortality and VFDs. Among a variety of clinical variables in this early timeframe, changes in oxygenation provided the most discernible predictor for outcomes in PARDS.

Several groups have demonstrated the discriminative ability of oxygenation metrics 24 hours after onset for outcomes in ARDS compared with those of metrics at onset $[8,13,14]$. Yehya et al. [13] reported that $\mathrm{PaO}_{2} / \mathrm{FiO}_{2}$ and OI, measured 24 hours after meeting ARDS criteria, accurately stratified the severity of lung injury. Parvathaneni et al. [14] reported up to $50 \%$ mortality in children who were categorized with severe ARDS at 24 hours. Although there was no difference in the discriminative power for mortality between PALICC criteria at diagnosis and 24 hours afterward, our findings revealed a significant association between initial progress within 24 hours and outcomes in PARDS. Subjects who showed identical or worsening severity within the first 24 hours exhibited an approximately three to four times higher risk for mortality and fewer VFDs. Moreover, those who were placed in the severe group at 24 hours demonstrated nearly $90 \%$ mortality, which implies a benefit for reclassification at 24 hours after PARDS diagnosis.

Although the cross-sectional nature of this study did not allow for determination of causality, the significant association of early progress in PARDS with final outcomes has critical implications. A possible hypothesis for this association is that differing responses to early phase resuscitation, including lung recruitment and subsequent improvement in ventilation/perfusion mismatches, might lead to different disease progression after onset. Indeed, the severity of PARDS changed widely in the first 24 hours, such that nearly two-thirds of patients were reclassified into categories of different severity. As there were no differences in these category changes in terms of the initial severity of PARDS, our results also highlight the importance of early interventions in the management of PARDS. Future prospective trials with larger cohorts are required to establish evidence-based interventions regarding populations who are most likely to benefit from changes in treatment within the first 24 hours.

Interestingly, we have shown that improvement in oxygenation was the most discernable predictor for outcomes in PARDS. Although changes in PIP, driving pressure, and PEEP also discriminated outcomes, $\Delta \mathrm{PaO}_{2} / \mathrm{FiO}_{2}$ demonstrated superior prognostic ability for mortality compared with the other variables. This finding is consistent with a recent report by Yehya and Thomas [19] that verified that $\mathrm{PaO}_{2} / \mathrm{FiO}_{2}$ at 24 hours and $\Delta \mathrm{PaO}_{2} / \mathrm{FiO}_{2}$ were exclusively associated with mortality, while pressure variables, including $\Delta \mathrm{PIP}, \Delta$ driving pressure, and $\triangle \mathrm{PEEP}$, were not. This distinguishing association between oxygenation response and survival indicates that oxygenation is a more appropriate surrogate metric for outcomes in PARDS compared with PIP or driving pressure. This finding raises questions regarding the beneficial effects of existing ventilation strategies in children that strictly limit TV and PIP $[15,20]$. 
Considering that the evidence for the effectiveness of low-TV ventilation in children has not been consistent [21], and that children exhibit a different respiratory physiology [5] that might result in dissimilar susceptibility to ventilator-induced lung injury compared to adults, detailed prospective trials are needed in children to identify the proper upper limits of TV, PIP, and driving pressure to achieve appropriate oxygenation.

In this study, PARDS occurred in approximately $26.2 \%$ of all ICU admissions of children. This prevalence was much higher than in previous reports [7]. Moreover, the overall mortality rate of $38.9 \%$ was higher than the rates of previous studies performed in North America [13,14,22] and Europe [8,23,24]. These discrepancies may result from differences in resources, socioeconomic circumstances, and patient characteristics. In our center, pediatric patients shared an ICU with adult patients until the year 2016, when the pediatric ICU was first established. For countries with limited resources, the indications for ICU admission are relatively more stringent than in more developed countries, which might lead to this increased incidence of PARDS in the ICU. Indeed, a recent meta-analysis demonstrated that studies performed in Asia had higher overall mortality than studies performed in Western countries [7]; the mortality result in our study is within the range of reported mortality in Asia [25-27]. In addition, postsurgical, cardiac, and neonatal cases are treated in separate specialized units in our center. As we have a large number of patients in the hematologyoncology and epilepsy centers, an increased incidence of these comorbidities might contribute to the increased mortality. In this study, logistic regression analysis demonstrated that mortality was significantly higher in subjects with those comorbidities (data not shown). Finally, subjects included in this study were confined to those who underwent at least one arterial blood-gas study; thus, mild cases could have been excluded. In this context, our findings demonstrate that PARDS remains a common cause of death in the field of pediatric critical care.

We acknowledge several limitations to this study. First, our study was conducted using a limited number of patients, since this was a single-center study. Second, treatment protocols regarding ventilator management might have varied over time. Third, our study results are limited to relatively short-term outcomes. In addition, since this study was retrospectively designed, we cannot exclude any possible bias regarding patient identification. Further prospective validations are needed to overcome the inherent limitations of this study design.

In conclusion, we demonstrated that the implementation of reclassification based on oxygenation metrics 24 hours after the initial diagnosis appropriately stratified outcomes in PARDS.
Progress within the first 24 hours was significantly associated with mortality and ventilator free days in PARDS. Among the variables evaluated during this early phase, oxygenation response was the most discernable surrogate metric for mortality. These results may provide a framework to design future interventional trials in PARDS and to optimize clinical practices.

\section{CONFLICT OF INTEREST}

No potential conflict of interest relevant to this article was reported.

\section{ACKNOWLEDGMENTS}

This work was supported by Institute for Information \& Communications Technology Promotion grant funded by the Korea Government (MSIT) (No. 2017-0-00599, Development of Big Data Analytics Platform for Military Health Information).

\section{ORCID}

Soo Yeon Kim https://orcid.org/0000-0003-4965-6193 Byuhree Kim https://orcid.org/0000-0001-6571-343X Sun Ha Choi https://orcid.org/0000-0003-4777-0187

Jong Deok Kim

In Suk Sol https://orcid.org/0000-0002-4266-5655 https://orcid.org/0000-0003-2470-9682

Min Jung Kim https://orcid.org/0000-0002-5634-9709

Yoon Hee Kim https://orcid.org/0000-0002-2149-8501

Kyung Won Kim Myung Hyun Sohn https://orcid.org/0000-0003-4529-6135 Kyu-Earn Kim https://orcid.org/0000-0002-2478-487X https://orcid.org/0000-0002-5730-3331

\section{SUPPLEMENTARY MATERIALS}

The online-only Supplement data are available with this article online: https://doi.org/10.4266/acc.2018.00136.

\section{REFERENCES}

1. Pfenninger J, Gerber A, Tschäppeler H, Zimmermann A. Adult respiratory distress syndrome in children. J Pediatr 1982;101: 352-7.

2. Bernard GR, Artigas A, Brigham KL, Carlet J, Falke K, Hudson $\mathrm{L}$, et al. The American-European Consensus Conference on ARDS: definitions, mechanisms, relevant outcomes, and clinical trial coordination. Am J Respir Crit Care Med 1994;149(3 Pt 1):818-24. 
3. ARDS Definition Task Force, Ranieri VM, Rubenfeld GD, Thompson BT, Ferguson ND, Caldwell E, et al. Acute respiratory distress syndrome: the Berlin Definition. JAMA 2012;307:2526-33.

4. Dahlem P, van Aalderen WM, Bos AP. Pediatric acute lung injury. Paediatr Respir Rev 2007;8:348-62.

5. Smith LS, Zimmerman JJ, Martin TR. Mechanisms of acute respiratory distress syndrome in children and adults: a review and suggestions for future research. Pediatr Crit Care Med 2013;14:631-43.

6. Khemani RG, Smith LS, Zimmerman JJ, Erickson S; Pediatric Acute Lung Injury Consensus Conference Group. Pediatric acute respiratory distress syndrome: definition, incidence, and epidemiology: proceedings from the Pediatric Acute Lung Injury Consensus Conference. Pediatr Crit Care Med 2015;16 (5 Suppl 1):S23-40.

7. Schouten LR, Veltkamp F, Bos AP, van Woensel JB, Serpa Neto A, Schultz MJ, et al. Incidence and mortality of acute respiratory distress syndrome in children: a systematic review and meta-analysis. Crit Care Med 2016;44:819-29.

8. López-Fernández Y, Azagra AM, de la Oliva P, Modesto V, Sánchez JI, Parrilla J, et al. Pediatric acute lung injury epidemiology and natural history study: incidence and outcome of the acute respiratory distress syndrome in children. Crit Care Med 2012;40:3238-45.

9. Wong JJ, Jit M, Sultana R, Mok YH, Yeo JG, Koh JW, et al. Mortality in pediatric acute respiratory distress syndrome: a systematic review and meta-analysis. J Intensive Care Med 2017 Jan 1 [Epub]. https://doi.org/10.1177/0885066617705109.

10. Villar J, Pérez-Méndez L, López J, Belda J, Blanco J, Saralegui I, et al. An early PEEP/FIO2 trial identifies different degrees of lung injury in patients with acute respiratory distress syndrome. Am J Respir Crit Care Med 2007;176:795-804.

11. Villar J, Pérez-Méndez L, Blanco J, Añón JM, Blanch L, Belda J, et al. A universal definition of ARDS: the $\mathrm{PaO} 2 / \mathrm{FiO} 2$ ratio under a standard ventilatory setting: a prospective, multicenter alidation study. Intensive Care Med 2013;39:583-92.

12. Trachsel D, McCrindle BW, Nakagawa S, Bohn D. Oxygenation index predicts outcome in children with acute hypoxemic respiratory failure. Am J Respir Crit Care Med 2005;172:206-11.

13. Yehya N, Servaes S, Thomas NJ. Characterizing degree of lung injury in pediatric acute respiratory distress syndrome. Crit Care Med 2015;43:937-46.

14. Parvathaneni K, Belani S, Leung D, Newth CJ, Khemani RG. Evaluating the performance of the pediatric acute lung injury consensus conference definition of acute respiratory distress syndrome. Pediatr Crit Care Med 2017;18:17-25.

15. Pediatric Acute Lung Injury Consensus Conference Group.
Pediatric acute respiratory distress syndrome: consensus recommendations from the Pediatric Acute Lung Injury Consensus Conference. Pediatr Crit Care Med 2015;16:428-39.

16. Khemani RG, Conti D, Alonzo TA, Bart RD 3rd, Newth CJ. Effect of tidal volume in children with acute hypoxemic respiratory failure. Intensive Care Med 2009;35:1428-37.

17. Amato MB, Meade MO, Slutsky AS, Brochard L, Costa EL, Schoenfeld DA, et al. Driving pressure and survival in the acute respiratory distress syndrome. N Engl J Med 2015;372:747-55.

18. Yehya N, Thomas NJ. Relevant outcomes in pediatric acute respiratory distress syndrome studies. Front Pediatr 2016;4:51.

19. Yehya N, Thomas NJ. Disassociating lung mechanics and oxygenation in pediatric acute respiratory distress syndrome. Crit Care Med 2017;45:1232-9.

20. Acute Respiratory Distress Syndrome Network, Brower RG, Matthay MA, Morris A, Schoenfeld D, Thompson BT, et al. Ventilation with lower tidal volumes as compared with traditional tidal volumes for acute lung injury and the acute respiratory distress syndrome. N Engl J Med 2000;342:1301-8.

21. de Jager P, Burgerhof JG, van Heerde M, Albers MJ, Markhorst DG, Kneyber MC. Tidal volume and mortality in mechanically ventilated children: a systematic review and meta-analysis of observational studies. Crit Care Med 2014;42:2461-72.

22. Zimmerman JJ, Akhtar SR, Caldwell E, Rubenfeld GD. Incidence and outcomes of pediatric acute lung injury. Pediatrics 2009;124:87-95.

23. Bindl L, Dresbach K, Lentze MJ. Incidence of acute respiratory distress syndrome in German children and adolescents: a population-based study. Crit Care Med 2005;33:209-312.

24. De Luca D, Piastra M, Chidini G, Tissieres P, Calderini E, Essouri $\mathrm{S}$, et al. The use of the Berlin definition for acute respiratory distress syndrome during infancy and early childhood: multicenter evaluation and expert consensus. Intensive Care Med 2013;39:2083-91.

25. Wong JJ, Phan HP, Phumeetham S, Ong JS, Chor YK, Qian S, et al. Risk stratification in pediatric acute respiratory distress syndrome: a multicenter observational study. Crit Care Med 2017;45:1820-8.

26. Wong JJ, Loh TF, Testoni D, Yeo JG, Mok YH, Lee JH. Epidemiology of pediatric acute respiratory distress syndrome in Singapore: risk factors and predictive respiratory indices for mortality. Front Pediatr 2014;2:78.

27. Hu X, Qian S, Xu F, Huang B, Zhou D, Wang Y, et al. Incidence, management and mortality of acute hypoxemic respiratory failure and acute respiratory distress syndrome from a prospective study of Chinese paediatric intensive care network. Acta Paediatr 2010;99:715-21. 

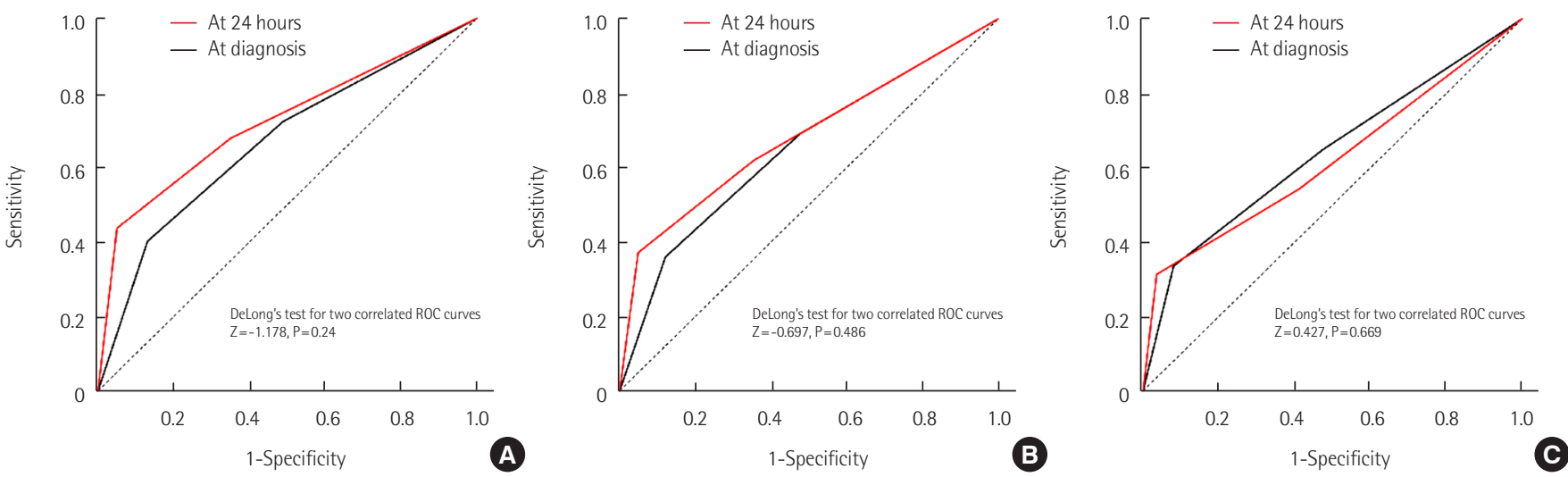

\begin{tabular}{lccc}
\hline & Area under the curve & $95 \% \mathrm{Cl}$ & P-value \\
\hline $\begin{array}{l}\text { (A) Mortality } \\
\text { At diagnosis }\end{array}$ & 0.668 & $0.607-0.729$ & $<0.001$ \\
At 24 hours & 0.724 & $0.655-0.792$ & $<0.001$ \\
(B) VFD 0 at day 28 & & & \\
At diagnosis & 0.651 & $0.592-0.710$ & $<0.001$ \\
At 24 hours & 0.683 & $0.615-0.752$ & $<0.001$ \\
(C) VFD $\leq 14$ at day 28 & & & $<0.001$ \\
At diagnosis & 0.641 & $0.582-0.699$ & $<0.001$ \\
At 24 hours & 0.620 & $0.548-0.693$ & \\
\hline
\end{tabular}

(D)

Supplementary Figure 1. Discriminative ability of Pediatric Acute Lung Injury Consensus Conference (PALICC) oxygenation categories at different time points for (A) mortality, (B) ventilator-free day (VFD) $=0$ at day 28 , and (C) VFD $\leq 14$ at day 28. (D) Receiver operating characteristic (ROC) curve analysis using PALICC categories at diagnosis, and at 24 hours afterward. Cl: confidence interval. 

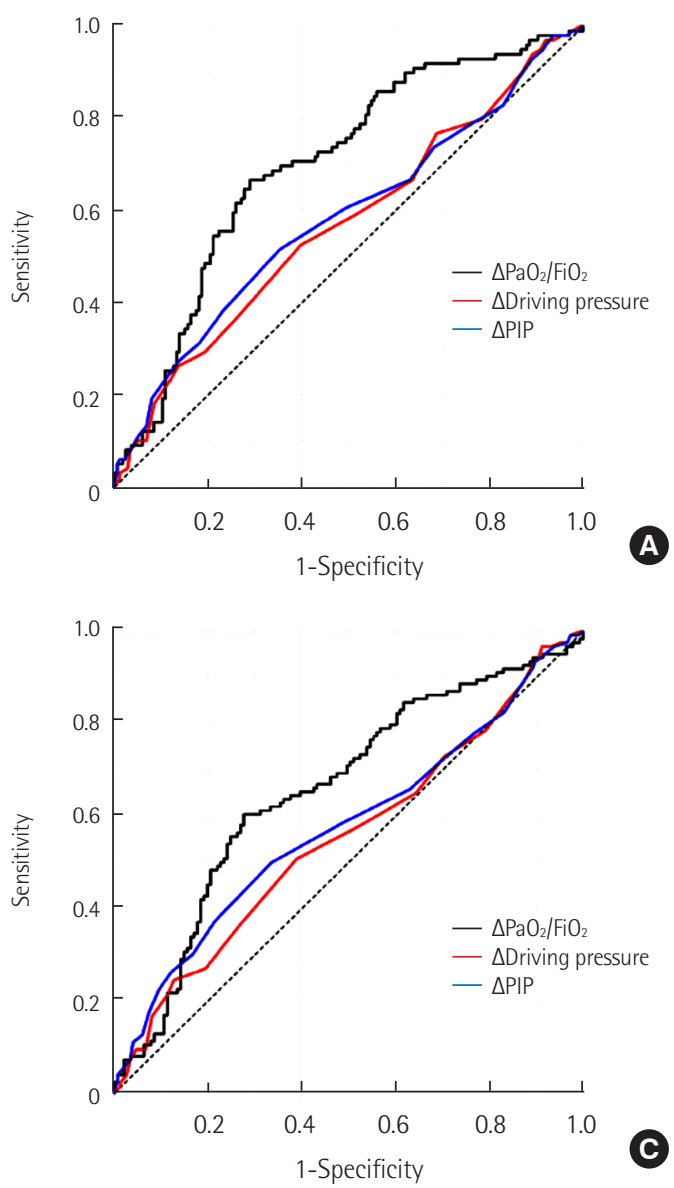
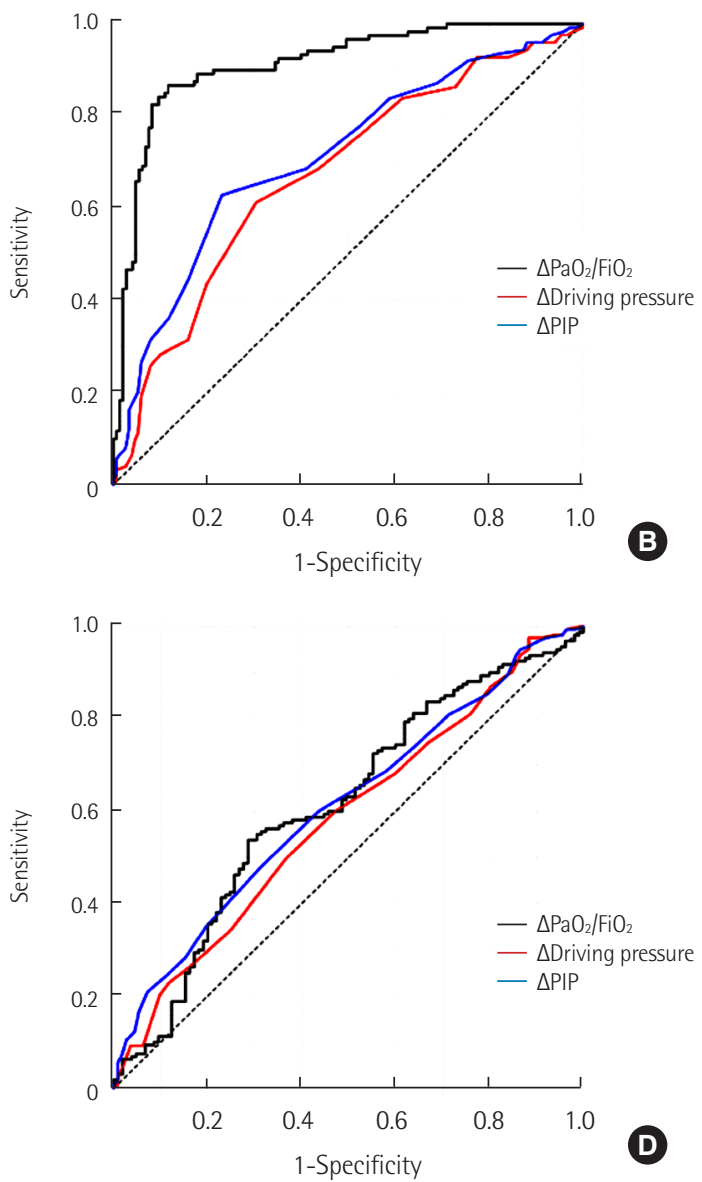

\begin{tabular}{|c|c|c|c|}
\hline & Area under the curve & $95 \% \mathrm{Cl}$ & P-value \\
\hline \multicolumn{4}{|l|}{ (A) Mortality } \\
\hline$\Delta \mathrm{PaO}_{2} / \mathrm{FiO}_{2}$ & 0.701 & $0.636-0.766$ & $<0.001$ \\
\hline$\Delta$ Driving pressure & 0.570 & $0.498-0.641$ & $<0.001$ \\
\hline$\Delta \mathrm{PIP}$ & 0.583 & $0.511-0.656$ & $<0.001$ \\
\hline \multicolumn{4}{|l|}{ (B) Initial progress } \\
\hline$\Delta \mathrm{PaO}_{2} / \mathrm{FiO}_{2}$ & 0.912 & $0.876-0.948$ & $<0.001$ \\
\hline$\Delta$ Driving pressure & 0.675 & $0.611-0.739$ & $<0.001$ \\
\hline$\Delta \mathrm{PIP}$ & 0.713 & $0.651-0.775$ & $<0.001$ \\
\hline \multicolumn{4}{|l|}{ (C) VFD $=0$ at day 28} \\
\hline$\Delta \mathrm{PaO}_{2} / \mathrm{FiO}_{2}$ & 0.658 & $0.592-0.725$ & $<0.001$ \\
\hline$\Delta$ Driving pressure & 0.554 & $0.486-0.623$ & $<0.001$ \\
\hline$\Delta \mathrm{PIP}$ & 0.578 & $0.509-0.646$ & $<0.001$ \\
\hline \multicolumn{4}{|l|}{ (D) VFD $\leq 14$ at day 28} \\
\hline$\Delta \mathrm{PaO}_{2} / \mathrm{FiO}_{2}$ & 0.613 & $0.543-0.683$ & $<0.001$ \\
\hline$\Delta$ Driving pressure & 0.584 & $0.516-0.652$ & $<0.001$ \\
\hline$\Delta \mathrm{PIP}$ & 0.611 & $0.545-0.678$ & $<0.001$ \\
\hline
\end{tabular}

E

Supplementary Figure 2. Discriminative ability of tested variables for (A) mortality, $(B)$ initial progress, $(C)$ ventilator-free day $(V F D)=0$ at day 28, and (D) VFD $\leq 14$ at day 28. (E) Receiver operating characteristic curve analysis using $\Delta \mathrm{PaO}_{2} / \mathrm{FiO}_{2}, \Delta$ driving pressure, and $\Delta \mathrm{PIP}$. $\Delta \mathrm{PaO}_{2} / \mathrm{FiO}_{2}$ : changes in arterial partial pressure of oxygen to fraction of inspired oxygen for the first 24 hours; $\Delta$ Driving pressure: changes in driving pressure for the first 24 hours; $\triangle \mathrm{PIP}$ : changes in peak inspiratory pressure for the first 24 hours; $\mathrm{Cl}$ : confidence interval. 